INSTITUT FÜR KLASSISCHE PHILOLOGIE, MITTEL- UND NEULATEIN

DER UNIVERSITÄT WIEN

ÖSTERREICHISCHE AKADEMIE DER WISSENSCHAFTEN

INSTITUT FÜR KULTURGESCHICHTE DER ANTIKE

CORPUS SCRIPTORUM ECCLESIASTICORUM LATINORUM

FACHBEREICH ALTERTUMSWISSENSCHAFT

UNIVERSITÄT SALZBURG

\title{
Wiener Studien
}

Zeitschrift für Klassische Philologie, Patristik

und lateinische Tradition

BAND 126

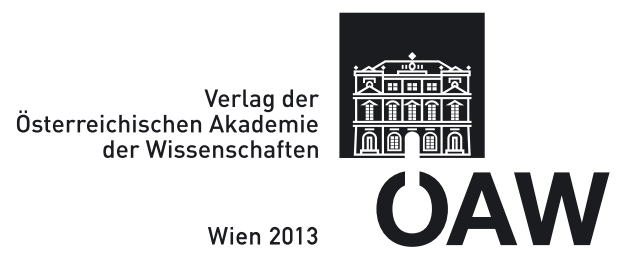




\title{
WIENER STUDIEN
}

Zeitschrift für Klassische Philologie, Patristik und lateinische Tradition

\author{
General Editor \\ Kurt Smolak (University of Vienna) \\ International Editorial Board \\ Herbert Bannert (University of Vienna) \\ Hildegund Müller (University of Notre Dame, Indiana) \\ Antonios Rengakos (Aristotle University of Thessaloniki) \\ Dirk Sacré (Catholic University of Leuven) \\ Danuta Shanzer (University of Vienna) \\ Ernst A. Schmidt (University of Tübingen) \\ Dorothea Weber (University of Salzburg) \\ Erich Woytek (University of Vienna)
}

Associate Editors

Stefan Büttner, Farouk F. Grewing, Elisabeth Klecker, Christine Harrauer, Christine Ratkowitsch, Franz Römer, Walter Stockert (University of Vienna)

Edited by

Institut für Klassische Philologie, Mittel- und Neulatein

der Universität Wien

Layout: Andrea Duchac

Manuscripts to be considered for publication should be sent to:

Wiener Studien

Institut für Klassische Philologie, Mittel- und Neulatein der Universität Wien, Universitätsring 1, A-1010 Wien.

Submitted manuscripts will be refereed by three of the editors.

Als internationale wissenschaftliche peer reviewed Zeitschrift von der ÖAW gefördert.

Die verwendete Papiersorte ist aus chlorfrei gebleichtem Zellstoff hergestellt, frei von säurebildenden Bestandteilen und alterungsbeständig.

Alle Rechte vorbehalten - All rights reserved

ISSN 0084-005X

ISBN 978-3-7001-7525-4

Copyright (C) 2012 by

Österreichische Akademie der Wissenschaften

Production: Prime Rate kft., Budapest

http://hw.oeaw.ac.at/7525-4

http://verlag.oeaw.ac.at 